\title{
A SET OF ALGORITHMS FOR THE INCOMPLETE GAMMA FUNCTIONS
}

\author{
N. M. TEMME \\ CWI, P.O. Box 94079 \\ 1090 GB Amsterdam, The Netherlands
}

\begin{abstract}
This paper gives fast and reliable algorithms for the numerical evaluation of the incomplete gamma functions and for auxiliary functions, such as functions related with the gamma function and error function. All these functions are of basic importance in applied probability problems.
\end{abstract}

\section{INTRODUCTION}

The incomplete gamma integral, and its equivalent, the chi-squared distribution, is of basic importance in applied probability problems arising in inventory, queueing, and reliability. The principle idea behind this paper is to make available reliable, fast, and simple algorithms with limited accuracy (say, nine significant digits) that are easily implemented by the practitioner in applied probability. This note intends to fulfill a long-felt need for such algorithms. We give a basic set of algorithms for computing the incomplete gamma integral, including programs for the gamma function and the error functions.

\section{AUXILIARY GAMMA FUNCTIONS}

The Euler gamma function is defined by

$$
\Gamma(x)=\int_{0}^{\infty} t^{x-1} e^{-t} d t, \quad x>0 .
$$


We consider positive values of $x$. We give approximations for $\Gamma(x)$ and for auxiliary functions. As a rule, we give nearly-best Chebyshev rational approximations for the functions. The rational approximations all give answers accurate to nine significant digits. The coefficients are given in Pascal programs and are computed by the Remes algorithm, with accuracy of 19 significant digits.

\subsection{The Function $\Gamma^{*}(x)$}

We need an algorithm for the "tempered" gamma function $\Gamma^{*}(x)$ defined by

$$
\Gamma(x)=\sqrt{2 \pi} e^{-x} x^{x-\frac{1}{2}} \Gamma^{*}(x), \quad x>0 .
$$

This function is of fundamental importance in algorithms when $x$ is large, because, on account of Stirling's formula, we have $\Gamma^{*}(x)=1+\mathcal{O}(1 / x)$, as $x \rightarrow \infty$. For instance, when ratios of gamma functions are used, it is allimportant to cancel the dominant parts in the fractions. The dominant part of the gamma function usually causes overflow, whereas the result of a combination of gamma functions may be representable.

For $x>1$, we use rational approximations; for $x \in(0,1)$, we use the recursion

$$
\Gamma^{*}(x)=e^{-1}\left(\frac{x+1}{x}\right)^{x+\frac{1}{2}} \Gamma^{*}(x+1) .
$$

In this way the algorithm is independent from other algorithms for the gamma function.

\subsection{Another Auxiliary Function for the Gamma Function}

In our algorithm for the incomplete gamma functions, we need the evaluation of $1 / \Gamma(1+x)-1$ for small values of $|x|$. In fact, we use the function $g(x)$ defined by

$$
\frac{1}{\Gamma(1+x)}=1+x(x-1) g(x), \quad 0 \leq x \leq 1 .
$$

This representation shows the vanishing of $1 / \Gamma(1+x)-1$ at $x=0, x=1$. On the interval $[0,1]$, the function $g(x)$ is computed by using a rational function. In the algorithm for the incomplete gamma function, we also need values on a neighboring interval, especially on $\left[1,1 \frac{1}{4}\right]$, which can be obtained by a simple stable recursion. For $x>2$, direct evaluation of $1 / \Gamma(1+x)-1$ is stable when the gamma function itself is used.

\subsection{The Gamma Function}

We use a rational approximation on the interval [2,3]. For $x \in(0,2)$ and $x \in$ $(3,10)$, we use recursion. For $x \geq 10$, we use the asymptotic expansion for the 
logarithm of the gamma function. We take care of large values, because overflow occurs very soon.

The special cases $x=n$ or $x=n+\frac{1}{2}, n$ integer, occur very frequently in practical problems and the gamma function values can be given very easily. For these reasons we treat these values separately.

\subsection{The Logarithm of the Gamma Function}

This function is not needed in our programs. We like to draw attention to a robust algorithm given in Macleod [5]. A program in Fortran gives nine significant decimals, with good care for underflow and overflow. Also, care is taken for the vanishing of $\ln \Gamma(x)$ at $x=1, x=2$. The algorithm is based on rational approximations, as given in Cody and Hillstrom [2]. For convenience we include a Pascal version of Macleod's algorithm in our collection.

Remark: $\Gamma(x)$ and $\ln \Gamma(x)$ may be obtained directly from $\Gamma^{*}(x)$. However, in that case the computation of the exponential and/or logarithmic function is needed, which may not be as fast as the direct evaluation by using rational approximations. Another point is that the special values of $\ln \Gamma(x)$ at $x=1$, $x=2$, will be inaccurate when taking the logarithm of $\Gamma(x)$.

\section{AUXILIARY ALGORITHMS FOR THE EXPONENTIAL AND LOGARITHMIC FUNCTIONS}

\subsection{The Function $\ln (1+x)-x$}

When we need $\ln (1+x)$ for small values of $|x|$, straightforward use of the standard log-function gives bad relative accuracy. For instance, when $|x|$ is so small that $1+x=1$ (on the computer), $\ln (1+x)$ is just equal to 0 , while we may need the correct value, which is of order $x+\mathcal{O}\left(x^{2}\right)$. In the case of the incomplete gamma functions, we need the evaluation of $\ln (1+x)-x$, which is of order $-\frac{1}{2} x^{2}+\vartheta\left(x^{3}\right)$ when $|x|$ is small. To obtain this quantity, we use a rational approximation on the interval $[0,1.36]$. On $[-0.70,0)$ we use a symmetry rule, namely, the relation

$$
\ln (1+x)-x=-[\ln (1+z)-z]+x z, \quad z=-\frac{x}{x+1} .
$$

\subsection{The Function $e^{x}-1$}

We also need an algorithm for the evaluation of $e^{x}-1$ for small values of $|x|$. This can be done by the Taylor series of $e^{x}$, but again we use rational approximations - in this case on the interval $\left[\ln \frac{1}{2}, \ln \frac{3}{2}\right]$. Note that in $\sinh x=$ $\left(e^{x}-e^{-x}\right) / 2$ also loss of accuracy occurs when $|x|$ is small. However, when we have $y=e^{x}-1$, a stable representation is $\sinh x=\frac{1}{2} y(y+2) /(y+1)$. 


\subsection{The Function $x^{a} e^{-x} / \Gamma(a+1)$}

The function

$$
D(a, x):=\frac{x^{a} e^{-x}}{\Gamma(a+1)}
$$

is the dominant part in many representations of the incomplete gamma functions. Especially when $a$ and $x$ are large, the computation of this quantity needs some care. We write $D(a, x)$ in the form

$$
D(a, x)=\frac{e^{-a[\mu-\ln (1+\mu)]}}{\sqrt{2 \pi a} \Gamma^{*}(a)}, \quad \mu=\frac{x-a}{a},
$$

where $\Gamma^{*}(x)$ is defined in Eq. (2.2). When $|\mu|$ is small, the computation of $\mu-\ln (1+\mu)$ in the exponential function should be done, for instance, by using the algorithm mentioned in Section 3.1. We want to point out that, for large parameters $a, x$, the accuracy in the computations of the incomplete gamma functions strongly depends on the evaluation of the function $D(a, x)$. Representation (3.1) does not solve all problems, because the well-known loss of accuracy in evaluating the exponential function for a large argument is still a crucial point.

\section{THE ERROR FUNCTIONS}

We define

$$
\operatorname{erf} z=\frac{2}{\sqrt{\pi}} \int_{0}^{z} e^{-t^{2}} d t, \quad \operatorname{erfc} z=1-\operatorname{erf} z=\frac{2}{\sqrt{\pi}} \int_{z}^{\infty} e^{-t^{2}} d t .
$$

These functions are used in statistics and probability theory as the normal distribution functions, with somewhat different notation. For instance, we have

$$
Z(x)=\frac{1}{\sqrt{2 \pi}} e^{-\frac{1}{2} x^{2}}, \quad P(x)=\int_{-\infty}^{x} Z(t) d t, \quad Q(x)=\int_{x}^{\infty} Z(t) d t .
$$

It easily follows that

$$
P(x)=\frac{1}{2} \operatorname{erfc}(-x / \sqrt{2}), \quad Q(x)=\frac{1}{2} \operatorname{erfc}(x / \sqrt{2}) .
$$

We have the symmetry relations

$$
\operatorname{erf}(-x)=-\operatorname{erf} x, \quad \operatorname{erfc}(-x)=2-\operatorname{erfc} x .
$$

The error functions are entire functions.

We base the algorithm on rational approximations of Cody [1]. The standard method for real $x$ is to compute directly erf $x$ and erfc $x$ indirectly (as $1-$ erf $x$ ) when erf $x$ is smaller in value. Otherwise, erfc $x$ is computed directly. We have erf $x_{0}=\operatorname{erfc} x_{0}$ when $x_{0} \cong 0.47$, and we take 0.50 as the changeover point. 
Another feature of our algorithm is that we can obtain $e^{x^{2}} \operatorname{erfc} x$ when $x$ is positive. This is of great importance in numerical algorithms, because the computation of erfc $x$ causes underflow for quite moderate values of $x$, due to the dominant term $e^{-x^{2}}$ in the asymptotic behavior of erfc $x$.

This all is handled in one master algorithm:

errorfunction (x: double; erfc, expo: boolean): double;

where the booleans erfc and expo mean the following:

- if $\operatorname{erfc}=$ true and $\operatorname{expo}=$ false then errorfunction computes erfc $x$;

- if erfc $=$ true and expo $=$ true and $x>0$ then errorfunction computes $e^{x^{2}}$ erfc $x ;$ and

- if $\operatorname{erfc}=$ false then errorfunction computes erf $x$.

In three special-purpose algorithms, the use of errorfunction is explained further. The rational approximations are accurate to nine significant decimals.

\section{THE INCOMPLETE GAMMA FUNCTIONS}

We define

$$
\gamma(a, x)=\int_{0}^{x} t^{a-1} e^{-t} d t, \quad \Gamma(a, x)=\int_{x}^{\infty} t^{a-1} e^{-t} d t .
$$

We assume that $a$ and $x$ are positive numbers. It is useful to define the normalizations

$$
P(a, x)=\frac{\gamma(a, x)}{\Gamma(a)}, \quad Q(a, x)=\frac{\Gamma(a, x)}{\Gamma(a)},
$$

of which the sum equals unity.

In statistics and probability theory, one is more familiar with the chisquared probability functions, which are defined by

$$
P\left(\chi^{2} \mid \nu\right)=P(a, x), \quad Q\left(\chi^{2} \mid \nu\right)=Q(a, x), \quad \nu=2 a, \quad \chi^{2}=2 x .
$$

In other words,

$$
\begin{aligned}
& P\left(\chi^{2} \mid \nu\right)=\frac{1}{2^{\nu / 2} \Gamma\left(\frac{1}{2} \nu\right)} \int_{0}^{\chi^{2}} t^{\nu / 2-1} e^{-t / 2} d t \\
& Q\left(\chi^{2} \mid \nu\right)=\frac{1}{2^{\nu / 2} \Gamma\left(\frac{1}{2} \nu\right)} \int_{\chi^{2}}^{\infty} t^{\nu / 2-1} e^{-t / 2} d t
\end{aligned}
$$

When $\nu$ is even, we have the Poisson distribution, which reads

$$
Q\left(\chi^{2} \mid \nu\right)=\sum_{j=0}^{c-1} e^{-m} \frac{m^{j}}{j !}, \quad c=\frac{1}{2} \nu, \quad m=\frac{1}{2} \chi^{2} .
$$




\subsection{Gautschi's Algorithm}

Our algorithm for the computation of $P(a, x), Q(a, x)$ is partly based on Gautschi [4]. Another interesting paper in the same spirit is DiDonato and Morris [3]. A rather short Fortran program appeared in the statistical literature in Shea [6], which also compared the results with Gautschi's Fortran program.

We will explain a few aspects of our algorithm. The quarter plane $\{a>0$, $x>0\}$ is divided in two parts, roughly by using the diagonal $a=x$ with small corrections at the origin. When $a \geq x, P(a, x)$ is computed directly, and $Q(a, x)$ indirectly as $1-P(a, x)$. When $x>a, Q(a, x)$ is computed directly.

$P(a, x)$ is computed by the Taylor series:

$$
P(a, x)=\frac{x^{a} e^{-x}}{\Gamma(a+1)} \sum_{n=0}^{\infty} x^{n} \frac{\Gamma(a+1)}{\Gamma(n+a+1)},
$$

which is stable and converges quite fast when $a \geq x$. When both parameters are large and of the same order, the convergence slows down. In that case we modify Gautschi's algorithm (see Section 5.2).

For small values of $x$, that is, when $x \leq 1, Q(a, x)$ is computed by using the representation

$$
Q(a, x)=1-\frac{x^{a}}{\Gamma(a)} \sum_{n=0}^{\infty} \frac{(-1)^{n} x^{n}}{(a+n) n !},
$$

in which a different Taylor expansion of $P(a, x)$ is used. The crucial part of the algorithm is the computation of $1-x^{a} / \Gamma(a+1)$ (obtained from the first term of the series). When $a$ is small, loss of accuracy may occur in the subtraction; also, when $x$ is near unity and $a$ is near zero or unity, instabilities occur. Writing

$$
1-\frac{x^{a}}{\Gamma(a+1)}=\left[1-\frac{1}{\Gamma(a+1)}\right]-\frac{\left[x^{a}-1\right]}{\Gamma(1+a)},
$$

we can control the instabilities by computing the terms between square brackets separately. For computing these terms, the auxiliary algorithms in the previous sections are used.

When $x>1, x>a$, the function $Q(a, x)$ is computed by using Legendre's continued fraction:

$$
Q(a, x)=\frac{x^{a} e^{-x} / \Gamma(a)}{x+\frac{1-a}{1+\frac{1}{x+\frac{2-a}{1+\frac{2}{x+\frac{3-a}{1+\ddots}}}}}}
$$


This expansion converges for all $x>0$ and any value of $a$ (also for complex values of $a$ and $x$ ). The continued fraction is evaluated by turning the fraction into a series, as described in Gautschi [4]. Convergence is fast when $x \gg a$. Again, convergence slows down when both $x$ and $a$ are large and of the same order.

\subsection{Evaluation Based on Uniform Expansions}

When $a$ is large, the functions $P(a, \lambda a), Q(a, \lambda a)$ change rapidly when $\lambda$ crosses the value 1 . This sudden change in behavior is the cause of bad convergence of standard expansions (5.5) and (5.6). The asymptotic behavior of the incomplete gamma functions can be described by using the error function (the normal distribution function). In Temme [7] uniform expansions of the incomplete gamma functions in terms of the error function are given; in Temme [8] a numerical algorithm is described based on the uniform representations. We summarize the basic elements of this algorithm.

Let $\eta$ be the real number defined by

$$
\frac{1}{2} \eta^{2}=\lambda-1-\ln \lambda, \quad \lambda>0, \quad \operatorname{sign}(\eta)=\operatorname{sign}(\lambda-1) .
$$

Then we write, with $\lambda=x / a$,

$$
\begin{aligned}
& Q(a, x)=\frac{1}{2} \operatorname{erfc}(\eta \sqrt{a / 2})+R_{a}(\eta), \\
& P(a, x)=\frac{1}{2} \operatorname{erfc}(-\eta \sqrt{a / 2})-R_{a}(\eta) .
\end{aligned}
$$

The error functions are the dominant terms that describe the transition at $a=$ $x$. Observe that the property $P+Q=1$ is reflected in the error functions, because $\operatorname{erfc} x+\operatorname{erfc}(-x)=1$, and in having the same remainder $R_{a}(\eta)$. We write

$$
R_{a}(\eta)=\frac{e^{-\frac{1}{2} a \eta^{2}}}{\sqrt{2 \pi a}} S_{a}(\eta) .
$$

From our papers already quoted, it follows that $S_{a}(\eta)$ is slowly varying for all $\eta \in \mathbb{R}$, that is, for all $\lambda \in(0, \infty)$, where $\lambda=x / a$. We expand

$$
S_{a}(\eta)=\frac{1}{\Gamma^{*}(a)} \sum_{m=0}^{\infty} b_{m}(a) \eta^{m} .
$$

In Temme [8] we showed that the coefficients $b_{m}$ can be computed by the recursion

$$
b_{m-1}(a)=f_{m}+\frac{m+1}{a} b_{m+1}(a),
$$

where the numbers $f_{m}$ are given by

$$
f_{0}=1, \quad f_{1}=-\frac{1}{3}, \quad f_{2}=\frac{1}{12}, \quad f_{3}=-\frac{2}{135}, \quad f_{4}=\frac{1}{864}, \quad f_{5}=\frac{1}{2835} .
$$

Further coefficients can be generated by the recursion 


$$
f_{m}=-\frac{m+1}{m+2}\left[\frac{m-1}{3 m} f_{m-1}+\sum_{j=3}^{m-1} \frac{f_{j-1} f_{m+1-j}}{m+2-j}\right], \quad m \geq 4 .
$$

The backward recursion for $b_{m}$ is stable; we can start with the false initial values

$$
b_{\nu}(a)=f_{\nu+1}, \quad b_{\nu-1}(a)=f_{\nu},
$$

for some value $\nu$. In the algorithm we use $\nu=14$. The backward recursion scheme converges better as $a$ increases. Here, "convergence" means that the computed values of $b_{m}(a)$ obtained by recursion (5.10), and needed in the series in Eq. (5.9), are accurate enough for obtaining the desired precision.

We want to obtain nine-digit accuracy, and we use Eq. (5.9) for $\eta \in$ $(-0.92,0.19)$. This corresponds with (about) $\lambda \in(0.8,1.2)$. Then, when we take $a \geq 25$ and only ten terms in the series of Eq. (5.9), we can obtain results that are accurate to nine decimal digits for the computation of $P(a, x), Q(a, x)$ by using the representations of Eq. (5.7).

\section{References}

1. Cody, W.J. (1969). Rational Chebyshev approximations for the error function. Mathematics of Computation 23: 631-637.

2. Cody, W.J. \& Hillstrom, K.E. (1967). Chebyshev approximations for the natural logarithm of the gamma function. Mathematics of Computation 21: 198-203.

3. DiDonato, A.R. \& Morris, A.H., Jr. (1986). Computation of the incomplete gamma functions. ACM Transactions on Mathematical Software 12: 377-393.

4. Gautschi, W. (1979). A computational procedure for incomplete gamma functions. ACM Transactions on Mathematical Software 5: 466-481.

5. Macleod, A.J. (1989). A robust and reliable algorithm for the logarithm of the gamma function; algorithm AS 245. Applied Statistics 38: 397-423.

6. Shea, B.L. (1988). Chi-squared and incomplete gamma integral. Applied Statistics 37: 466-473.

7. Temme, N.M. (1979). The asymptotic expansions of the incomplete gamma functions. SIAM Journal on Mathematical Analysis 10: 239-253.

8. Temme, N.M. (1987). On the computation of the incomplete gamma functions for large values of the parameters. In E.J.C. Mason \& M.G. Cox (eds.), Algorithms for approximation. Proceedings of the IMA-Conference, Shrivenham, July 15-19, 1985, Oxford, Clarendon, pp. 479-489. 


\section{APPENDIX: PASCAL PROGRAMS}

The Pascal programs were designed on a Macintosh in Think Pascal. No special features of this compiler are used; units are not used. We expect that the program will run on many other compilers and that no significant changes are needed.

The user has to specify three constants:

- machtol is the machine precision: the smallest constant such that $1 \pm$ machtol is different from unity.

- giant is the upper limit of machine-representable numbers (the "overflow" bound).

- $d$ warf is the lower limit of machine-representable numbers (the "underflow" bound).

Of course, these numbers may be slightly larger, smaller, and larger, respectively, than the exact machine constants of the user's computer.

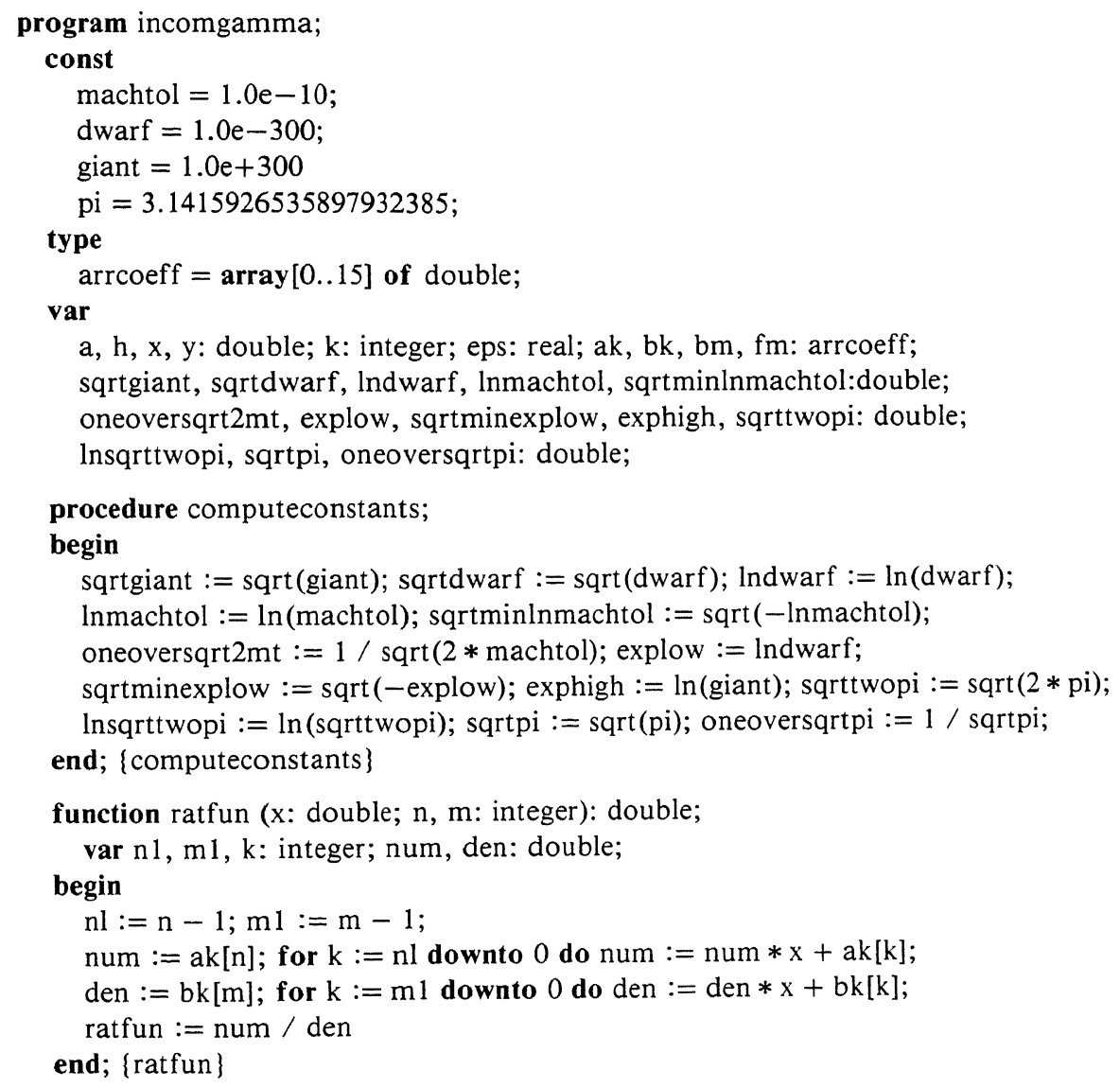


function exmin 1 (x: double): double;

var y: double;

begin

if $\mathrm{x}<\operatorname{lnmachtol}$ then $\mathrm{y}:=-1$ else if $\mathrm{x}>$ exphigh then $\mathrm{y}:=$ giant else

if $(x<-0.69)$ or $(x>0.41)$ then $y:=\exp (x)-1.0$ else

if $\operatorname{abs}(\mathrm{x})<$ machtol then $\mathrm{y}:=\mathrm{x}$ else

begin

$$
\begin{array}{rlrl}
\operatorname{ak}[0]:=9.999999998390 \mathrm{e}-1 ; & & \text { bk }[0]:=1.000000000000 \mathrm{e}+0 \\
\operatorname{ak}[1]:=6.652950247674 \mathrm{e}-2 ; & & \text { bk }[1]:=-4.334704979491 \mathrm{e}-1 ; \\
\operatorname{ak}[2]:=2.331217139081 \mathrm{e}-2 ; & & \text { bk }[2]:=7.338073943202 \mathrm{e}-2 ; \\
\text { ak[3] }:=1.107965764952 \mathrm{e}-3 ; & & \text { bk }[3]:=-5.003986850699 \mathrm{e}-3 ; \\
\mathrm{y}:=\mathrm{x} * \text { ratfun }(\mathrm{x}, 3,3) &
\end{array}
$$

end;

exmin $1:=y$

end; $\{$ exmin 1$\}$

function auxln (x: double): double;

var $y, z$ : double; $n$ : integer;

begin

if $x \leqq-1$ then $y:=-$ giant else if $(x<-0.70)$ or $(x>1.36)$ then $y:=\ln (1+x)-x$

else if $\operatorname{abs}(x)<$ machtol then $y:=-0.5 * \operatorname{sqr}(x)$ else

begin

$\mathrm{ak}[0]:=-4.999999994526 \mathrm{e}-1 ; \quad$ bk $[0]:=1.000000000000 \mathrm{e}+0 ;$

$\operatorname{ak}[1]:=-5.717084236157 \mathrm{e}-1 ; \quad$ bk $[1]:=1.810083408290 \mathrm{e}+0$;

$\operatorname{ak}[2]:=-1.423751838241 \mathrm{e}-1 ; \quad$ bk[2] $:=9.914744762863 \mathrm{e}-1$;

$\operatorname{ak}[3]:=-8.310525299547 \mathrm{e}-4 ; \quad$ bk $[3]:=1.575899184525 \mathrm{e}-1$;

$\operatorname{ak}[4]:=3.899341537646 \mathrm{e}-5$;

if $\mathrm{x}>0$ then $\mathrm{y}:=\operatorname{sqr}(\mathrm{x}) * \operatorname{ratfun}(\mathrm{x}, 4,3)$ else

begin

$z:=-x /(1+x) ;$ if $z>1.36$ then $y:=-(\ln (1+z)-z)+x * z$

else $y:=-\operatorname{sqr}(z) * \operatorname{ratfun}(z, 4,3)+x * z$

end

end;

auxln $:=y$

end; $\{$ auxln $\}$

function gammastar ( $\mathrm{x}$ : double): double;

var $a, g, s$ : double; $j, k$ : integer;

begin

if $\mathrm{x}>1.0 \mathrm{e} 10$ then

begin if $x>1 /(12 *$ machtol $)$ then $g:=1.0$ else $g:=1.0+1 /(12 * x)$

end else if $x \geqq 12.0$ then

begin

$$
\begin{array}{lll}
\operatorname{ak}[0]:=1.000000000949 \mathrm{e}+0 ; & \mathrm{bk}[0]:=1.000000000000 \mathrm{e}+0 ; \\
\operatorname{ak}[1]:=9.781658613041 \mathrm{e}-1 ; & \mathrm{bk}[1]:=8.948328926305 \mathrm{e}-1 ; \\
\operatorname{ak}[2]:=7.806359425652 \mathrm{e}-2 ; &
\end{array}
$$

end else if $x \geqq 1.0$ then 
begin

$\operatorname{ak}[0]:=5.115471897484 \mathrm{e}-2$;

$\mathrm{bk}[0]:=1.544892866413 \mathrm{e}-2$;

$\operatorname{ak}[1]:=4.990196893575 \mathrm{e}-1$;

$\mathrm{bk}[1]:=4.241288251916 \mathrm{e}-1$;

$\operatorname{ak}[2]:=9.404953102900 \mathrm{e}-1$;

$\mathrm{bk}[2]:=8.571609363101 \mathrm{e}-1$

$\operatorname{ak}[3]:=9.999999625957 \mathrm{e}-1$;

$\mathrm{bk}[3]:=1.000000000000 \mathrm{e}+0$;

$\mathrm{g}:=\operatorname{ratfun}(\mathrm{x}, 3,3)$

end else if $x>$ dwarf then

begin

$\mathrm{a}:=1.0+1.0 / \mathrm{x} ; \mathrm{g}:=\operatorname{gammastar}(\mathrm{x}+1) * \operatorname{sqrt}(\mathrm{a}) * \exp (-1.0+\mathrm{x} * \ln (\mathrm{a}))$

end else $g:=1.0 /$ (sqrttwopi * sqrtdwarf);

gammastar :=g;

end; \{gammastar\}

function gamma ( $\mathrm{x}$ : double): double;

var $a, g, s, d w$ : double; j, k, kl, m: integer;

begin

if $x \leqq d w a r f$ then $g:=1.0 /$ dwarf else

begin

$\mathrm{k}:=\operatorname{round}(\mathrm{x}) ; \mathrm{m}:=\operatorname{trunc}(\mathrm{x}) ; \mathrm{k} 1:=\mathrm{k}-1$;

if $\mathrm{k}=0$ then $\mathrm{dw}:=\mathrm{dwarf}$ else $\mathrm{dw}:=(1.0+\mathrm{x}) *$ machtol;

if $(a b s(k-x)<d w)$ and $(x \leqq 15)$ then

begin $\mathrm{g}:=1.0$; for $\mathrm{j}:=2$ to $\mathrm{k} 1 \mathrm{do} g:=\mathrm{g} * \mathrm{j}$ end

else if $(a b s((x-m)-0.5)<(1.0+x) *$ machtol $)$ and $(x \leqq 15)$ then

begin $g:=$ sqrtpi; for $j:=1$ to $m$ do $g:=g *(j-0.5)$ end

else

begin

$\operatorname{ak}[0]:=1.000000000000 \mathrm{e}+0 ; \quad$ bk $[0]:=-1.345271397926 \mathrm{e}-1$;

$\operatorname{ak}[1]:=-3.965937302325 \mathrm{e}-1 ; \quad$ bk $[1]:=1.510518912977 \mathrm{e}+0 ;$

$\operatorname{ak}[2]:=2.546766167439 \mathrm{e}-1 ; \quad$ bk $[2]:=-6.508685450017 \mathrm{e}-1$;

$\operatorname{ak}[3]:=-4.880928874015 \mathrm{e}-2 ; \quad$ bk $[3]:=9.766752854610 \mathrm{e}-2$

$\mathrm{ak}[4]:=9.308302710346 \mathrm{e}-3 ; \quad$ bk $[4]:=-5.024949667262 \mathrm{e}-3$;

if $x<1.0$ then $g:=\operatorname{ratfun}(x+2,4,4) /(x *(x+1.0))$

else if $x<2$ then $g:=\operatorname{ratfun}(x+1.0,4,4) / x$

else if $x<3$ then $g:=\operatorname{ratfun}(x, 4,4)$

else if $x<10$ then

begin $\mathrm{g}:=1.0 ; \mathrm{a}:=\mathrm{x}$;

repeat $\mathrm{a}:=\mathrm{a}-1.0 ; \mathrm{g}:=\mathrm{a} * \mathrm{~g}$ until $\mathrm{a}<3$;

$\mathrm{g}:=\mathrm{g} * \operatorname{ratfun}(\mathrm{a}, 4,4)$

end else if $x<$ exphigh then

begin

$\mathrm{a}:=1.0 / \operatorname{sqr}(\mathrm{x})$;

$\mathrm{g}:=(1.0+\mathrm{a} *(-3.33333333333 \mathrm{e}-2+\mathrm{a} * 9.52380952381 \mathrm{e}-3)) /$

$(12.0 * x) ; \mathrm{a}:=-\mathrm{x}+(\mathrm{x}-0.5) * \ln (\mathrm{x})+\mathrm{g}+\operatorname{lnsqrttwopi}$;

if $\mathrm{a}<\operatorname{exphigh}$ then $\mathrm{g}:=\exp (\mathrm{a})$ else $\mathrm{g}:=$ giant;

end else $g:=$ giant

end;

gamma $:=\mathrm{g}$

end; \{gamma\} 
function auxgam (x: double): double;

$\{$ function $g(x)$ in $1 / \Gamma(1+x)=1+x *(x-1) * g(x), 0 \leqq x \leqq 1.0\}$

var $g$ : double;

begin

$$
\begin{array}{ll}
\operatorname{ak}[0]:=-5.772156647338 \mathrm{e}-1 ; & \mathrm{bk}[0]:=1.000000000000 \mathrm{e}+0 \\
\operatorname{ak}[1]:=-1.087824060619 \mathrm{e}-1 ; & \mathrm{bk}[1]:=3.247396119172 \mathrm{e}-1 ; \\
\operatorname{ak}[2]:=4.369287357367 \mathrm{e}-2 ; & \mathrm{bk}[2]:=1.776068284106 \mathrm{e}-1 ; \\
\operatorname{ak}[3]:=-6.127046810372 \mathrm{e}-3 ; & \mathrm{bk}[3]:=2.322361333467 \mathrm{e}-2 ; \\
& \mathrm{bk}[4]:=8.148654046054 \mathrm{e}-3
\end{array}
$$

if $x \leqq-1.0$ then $g:=-0.5$ else if $x<0$ then

$$
\mathrm{g}:=-(1.0+\operatorname{sqr}(\mathrm{x}+1.0) * \operatorname{ratfun}(\mathrm{x}+1.0,3,4)) /(1.0-\mathrm{x})
$$

else if $x \leqq 1.0$ then $g:=\operatorname{ratfun}(x, 3,4)$

else if $x \leqq 2.0$ then $g:=((x-2.0) * \operatorname{ratfun}(x-1,3,4)-1.0) / \operatorname{sqr}(x)$

else $g:=(1 / \operatorname{gamma}(x+1.0)-1) /(x *(x-1.0))$;

auxgam $:=\mathrm{g}$

end; \{auxgam $\}$

function lngamma (x: double): double;

$\{\ln \Gamma(x)$; rational approximations from Cody \& Hillstrom (1967)\}

var $a, g, y$ : double;

begin if $x>12$ then

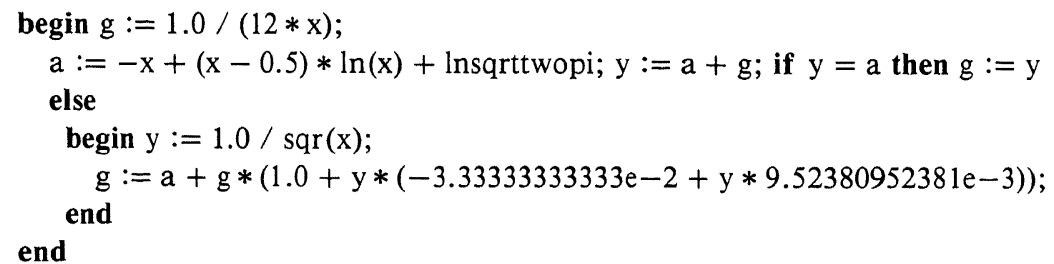

else if $x \geqq 4$ then

begin

$\operatorname{ak}[0]:=-2.12159572323 \mathrm{e} 5 ; \quad b k[0]:=-1.16328495004 \mathrm{e} 5 ;$

$\operatorname{ak}[1]:=2.30661510616 \mathrm{e} 5 ; \quad \quad b k[1]:=-1.46025937511 \mathrm{e} 5 ;$

$\operatorname{ak}[2]:=2.74647644705 \mathrm{e} 4 ; \quad$ bk[2] $:=-2.42357409629 \mathrm{e}$;

$\operatorname{ak}[3]:=-4.02621119975 \mathrm{e} 4 ; \quad \operatorname{bk}[3]:=-5.70691009324 \mathrm{e} 2 ;$

$\operatorname{ak}[4]:=-2.29660729780 \mathrm{e} 3 ; \quad b k[4]:=1.00000000000$;

$\mathrm{g}:=\operatorname{ratfun}(\mathrm{x}, 4,4)$

end

else if $x>1.5$ then

begin

$\operatorname{ak}[0]:=-7.83359299449 \mathrm{e} 1 ; \quad b k[0]:=4.70668766060 \mathrm{e} 1 ;$

$\operatorname{ak}[1]:=-1.42046296688 \mathrm{e} 2 ; \quad \operatorname{bk}[1]:=3.13399215894 \mathrm{e} 2 ;$

$\operatorname{ak}[2]:=1.37519416416 \mathrm{e} 2 ; \quad \operatorname{bk}[2]:=2.63505074721 \mathrm{e} 2 ;$

$\operatorname{ak}[3]:=7.86994924154 \mathrm{e} 1 ; \quad b k[3]:=4.33400022514 \mathrm{e} 1 ;$

$\operatorname{ak}[4]:=4.16438922228 ; \quad \operatorname{bk}[4]:=1.00000000000$;

end

$\mathrm{g}:=(\mathrm{x}-2) * \operatorname{ratfun}(\mathrm{x}, 4,4)$

else if $x>0$ then 


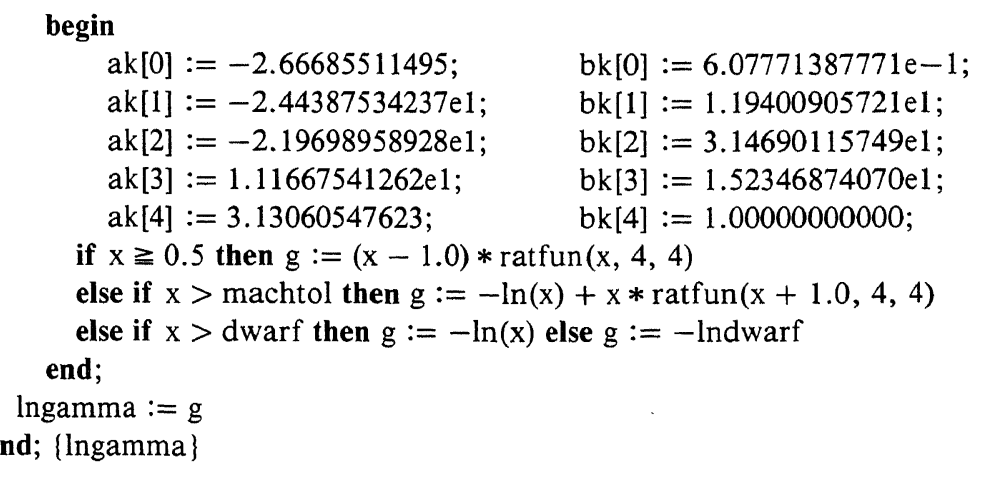

function errorfunction (x: double; erfc, expo: boolean): double;

\{rational approximations from Cody (1969)\}

var $\mathrm{xl}, \mathrm{y}, \mathrm{z}$ : double; done: boolean;

begin \{main of errorfunction\}

if erfc then

begin

if $\mathrm{x}<-$ sqrtminlnmachtol then $\mathrm{y}:=2$ else if $\mathrm{x}<-$ machtol then

$y:=2-$ errorfunction $(-x$, true, false) else if $x<$ machtol then $y:=1.0$

else if $x<0.5$ then

begin

if expo then $y:=\exp (x * x)$ else $y:=1.0$;

$y:=y *(1.0-$ errorfunction $(x$, false, false $))$

end

else if $x<4$ then

begin if expo then $y:=1.0$ else $y:=\exp (-x * x)$;

$$
\begin{array}{ll}
\operatorname{ak}[0]:=7.3738883116 ; & \operatorname{bk}[0]:=7.3739608908 ; \\
\operatorname{ak}[1]:=6.8650184849 ; & \operatorname{bk}[1]:=1.5184908190 \mathrm{e} ; \\
\operatorname{ak}[2]:=3.0317993362 ; & \operatorname{bk}[2]:=1.2795529509 \mathrm{e} 1 ; \\
\operatorname{ak}[3]:=5.6316961891 \mathrm{e}-1 ; & \mathrm{bk}[3]:=5.3542167949 ; \\
\operatorname{ak}[4]:=4.3187787405 \mathrm{e}-5 ; & \mathrm{bk}[4]:=1.0000000000 ;
\end{array}
$$

end

else

begin done $:=$ false; if expo then

begin $\mathrm{xl}:=1 /(\mathrm{dwarf} *$ sqrtpi);

if $x>x l$ then begin $y:=0.0$; done $:=$ true end

else if $x>$ oneoversqrt $2 \mathrm{mt}$ then

begin $y:=1.0 /(x *$ sqrtpi $)$; done $:=$ true end

else begin $z:=x * x ; y:=1.0$ end

end

else

begin $x l:=$ sqrtminexplow; if $x<x l$ then

begin $z:=x * x ; y:=\exp (-z)$;

if $\mathrm{x} * \mathrm{dwarf}>\mathrm{y} *$ oneoversqrtpi then

begin $y:=0$; done $:=$ true end 


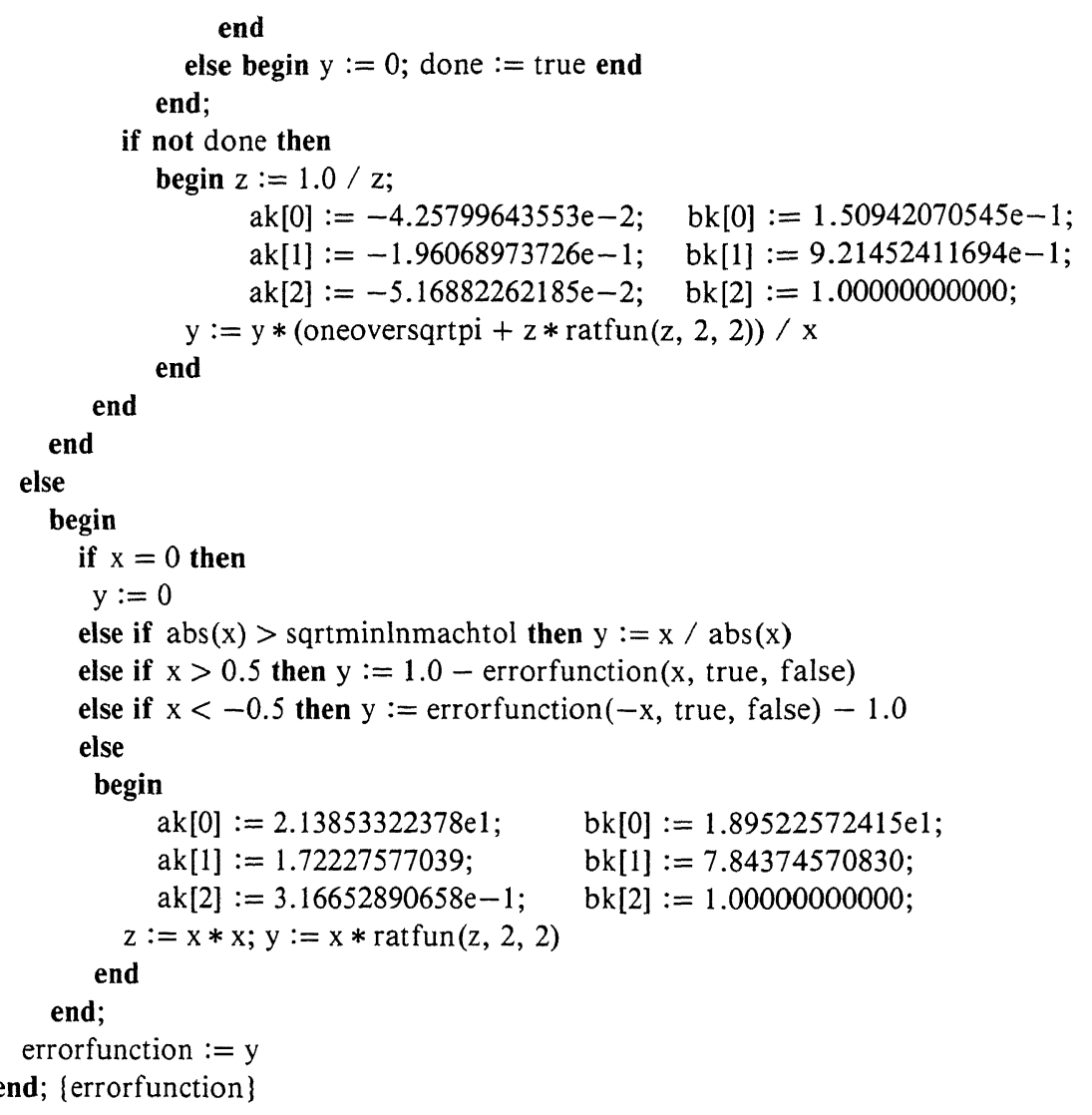

function erf (x: double): double;

begin erf := errorfunction( $x$, false, false) end; $\{$ erf $\}$

function erfc ( $\mathrm{x}$ : double): double;

begin erfc := errorfunction( $x$, true, false) end; $\{\operatorname{erfc}\}$

function erfctamed ( $x$ : double): double;

begin erfctamed :=errorfunction( $x$, true, true) end; (erfctamed

procedure incomgam (a, $\mathrm{x}$ : double; var $\mathrm{p}, \mathrm{q}$ : double; eps: real);

var $\mathrm{dp}, \ln x, \mathrm{mu}$, auxlnmu: double;

function alfa ( $x$ : double): double;

begin if $x>0.25$ then alfa $:=x+0.25$ else if $x \geq d w a r f$

then alfa $:=-0.6931 / \ln x$ else alfa $:=-0.6931 / \operatorname{lndwarf}$

end; $\{$ alpha $\}$

function dax: double;

begin

$\mathrm{mu}:=(\mathrm{x}-\mathrm{a}) / \mathrm{a} ;$ auxlnmu $:=\operatorname{aux} \ln (\mathrm{mu}) ; \mathrm{dp}:=\mathrm{a} *$ auxlnmu $-0.5 * \ln (2 * \mathrm{pi} * \mathrm{a}) ;$

if $\mathrm{dp}<$ explow then 


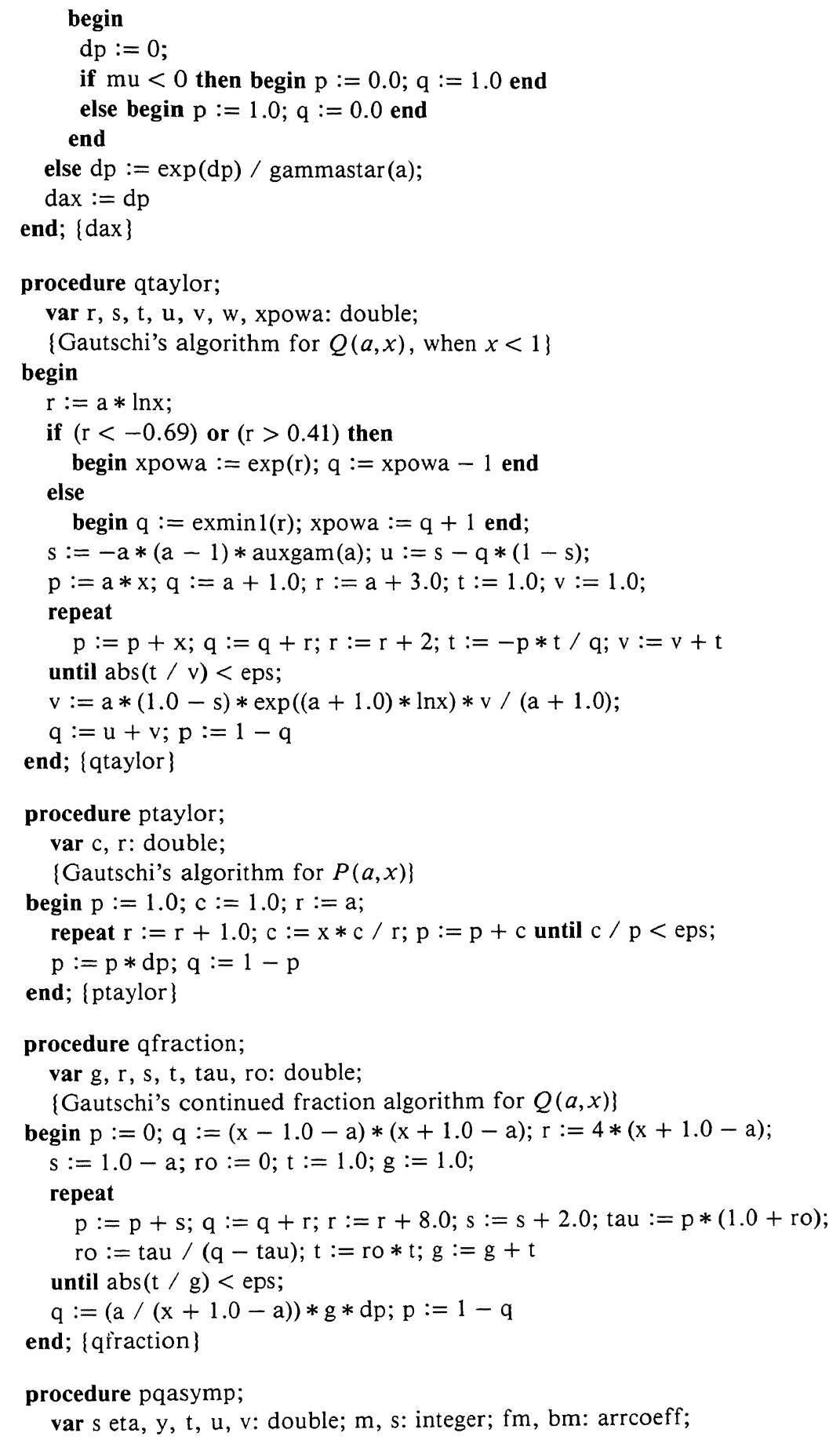


begin

fm $[0]:=1.0000000000 \mathrm{e}+0 ; \quad$ fm $[1]:=-3.3333333333 \mathrm{e}-1 ;$

$\mathrm{fm}[2]:=8.3333333333 \mathrm{e}-2 ; \quad \mathrm{fm}[3]:=-1.4814814815 \mathrm{e}-2$;

$\mathrm{fm}[4]:=1.1574074074 \mathrm{e}-3 ; \quad \mathrm{fm}[5]:=3.5273368607 \mathrm{e}-4$;

$\mathrm{fm}[6]:=-1.7875514403 \mathrm{e}-4 ; \quad \mathrm{fm}[7]:=3.9192631785 \mathrm{e}-5$;

fm $[8]:=-2.1854485107 e-6 ; \quad$ fm $[9]:=-1.8540622107 e-6$;

$\mathrm{fm}[10]:=8.2967113410 \mathrm{e}-7 ; \quad \mathrm{fm}[11]:=-1.7665952737 \mathrm{e}-7$;

$\mathrm{fm}[12]:=6.7078535434 \mathrm{e}-9 ; \quad \mathrm{fm}[13]:=1.0261809784 \mathrm{e}-8$;

$\operatorname{fm}[14]:=-4.3820360185 \mathrm{e}-9 ; \quad \mathrm{fm}[15]:=9.1476995822 \mathrm{e}-10 ;$

$\mathrm{y}:=-$ auxlnmu; eta $:=\operatorname{sqrt}(2 * \mathrm{y})$

$\mathrm{v}:=0.5 *$ errorfunction(sqrt $(\mathrm{a} * \mathrm{y})$, true, true) $* \operatorname{gammastar}(\mathrm{a}) * \operatorname{sqrt}(2 * \mathrm{pi} * \mathrm{a})$;

$\mathrm{s}:=1$; if $\mathrm{mu}<0$ then $\mathrm{s}:=-1$; eta $:=\mathrm{s} *$ eta;

$\operatorname{bm}[14]:=\mathrm{fm}[15] ; \operatorname{bm}[13]:=\mathrm{fm}[14] ; \mathrm{u}:=0$;

for $m:=13$ downto 1 do

begin

$\mathrm{t}:=\mathrm{fm}[\mathrm{m}]+(\mathrm{m}+1) * \mathrm{bm}[\mathrm{m}+1] / \mathrm{a} ; \mathrm{u}:=$ eta $* \mathrm{u}+\mathrm{t} ; \mathrm{bm}[\mathrm{m}-1]:=\mathrm{t} ;$

end; $u:=s * u ; p:=(u+v) * d p$

if $s=1$ then begin $q:=p ; p:=1.0-q$ end else $q:=1.0-p$

end; \{pqasymp \}

begin (main of incomgam\}

if $(a=0.0)$ and $(x=0.0)$ then begin $p:=0.5 ; q:=0.5$ end

else if $x=0.0$ then begin $p:=0.0 ; q:=1.0$ end

else if $a=0.0$ then begin $p:=1.0 ; q:=0.0$ end

else

begin

if $x \leqq d w a r f$ then $\ln x:=\operatorname{lndwarf}$ else $\ln x:=\ln (x)$;

$\mathrm{dp}:=\mathrm{dax} ;$ if $\mathrm{dp} \geqq$ dwarf then

begin

if $(a>25.0)$ and $(a b s(m u)<0.2)$ then pqasymp else

if $a>\operatorname{alfa}(x)$ then ptaylor else

begin if $x<1.0$ then qtaylor else qfraction end

end

end else begin if $a>x$ then $p:=0.0$ else $p:=1.0 ; q:=1.0-p$ end

end; \{incomgam\}

function checkincgam (a, $x$ : double; eps: real): double;

\{checks the relative accuracy in the recursions

$\left\{Q(a+1, x)=Q(a, x)+x^{a} * \exp (-x) / \Gamma(a+1)\right\}$

$\left\{P(a+1, x)=P(a, x)-x^{a} * \exp (-x) / \Gamma(a+1)\right\}$

$\operatorname{var} \mathrm{dp}, \mathrm{p}, \mathrm{q}, \mathrm{p} 1, \mathrm{q} 1, \mathrm{mu}, \mathrm{y}$ : double;

begin $\mathrm{mu}:=(\mathrm{x}-\mathrm{a}) / \mathrm{a} ; \mathrm{dp}:=\mathrm{a} * \operatorname{aux} \ln (\mathrm{mu})-0.5 * \ln (2 * \mathrm{pi} * \mathrm{a})$;

if $d p<\operatorname{explow}$ then $d p:=0$ else $d p:=\exp (d p) /$ gammastar(a);

incomgam $(a+1, x, p 1, q 1$, eps); incomgam $(a, x, p, q, e p s)$;

if $\mathrm{dp}>0$ then

begin

if $x>a$ then $y:=q 1 /(q+d p)-1$ else $y:=(p 1+d p) / p-1$

end else $y:=0$; 
checkincgam: $=\mathrm{y}$ end; \{checkincgam\}

begin \{main\} computeconstants; eps $:=1.0 \mathrm{e}-10 ; \mathrm{k}:=0 ; \mathrm{h}:=0$;

repeat $\{$ random returns an integer in the range $[-32768,32767]\}$

$\mathrm{a}:=$ machtol + abs(random $/ 300)$;

$\mathrm{x}:=$ machtol + abs(random $/ 320)$;

$y:=\operatorname{abs}(\operatorname{checkincgam}(a, x$, eps)); $\mathrm{k}:=\mathrm{k}+1$;

if $y>h$ then begin $h:=y$; writeln $\left(a: 16, x: 16, h,{ }^{\prime}, k\right)$ end; until $\mathrm{k}=1000$;

end. 